\title{
Auditory motion aftereffects
}

\author{
D. WESLEY GRANTHAM and FREDERIC L. WIGHTMAN \\ Northwestern University, Evanston, Illinois 60201
}

\begin{abstract}
Observers were adapted to simulated auditory movement produced by dynamically varying the interaural time and intensity differences of tones $(500$ or $2,000 \mathrm{~Hz})$ presented through headphones. At 10-sec intervals during adaptation, various probe tones were presented for $1 \mathrm{sec}$ (the frequency of the probe was always the same as that of the adaptation stimulus). Observers judged the direction of apparent movement ("left" or "right") of each probe tone. At $500 \mathrm{~Hz}$, with a 200-deg/sec adaptation velocity, "stationary" probe tones were consistently judged to move in the direction opposite to that of the adaptation stimulus. We call this result an auditory motion aftereffect. In slower velocity adaptation conditions, progressively less aftereffect was demonstrated. In the higher frequency condition $(2,000 \mathrm{~Hz}, 200$-deg/sec adaptation velocity), we found no evidence of motion aftereffect. The data are discussed in relation to the well-known visual analog-the "waterfall effect." Although the auditory aftereffect is weaker than the visual analog, the data suggest that auditory motion perception might be mediated, as is generally believed for the visual system, by direction-specific movement analyzers.
\end{abstract}

If one gazes at a continuously moving visual pattern for $30 \mathrm{sec}$ or more, then shifts one's gaze to a stationary visual pattern, one will typically report a strong perception of motion in the direction opposite to that of the original movement. This type of motion aftereffect is experienced often in everyday life and can be quite compelling. Laboratory investigation of this and related phenomena (e.g., the "waterfall" and spiral aftereffects) has been extensive and has spanned more than a century (see Wohlgemuth, 1911, and Gates, 1934, for reviews of the early work on visual aftereffects).

The generally accepted explanation for visual motion aftereffects is based on the presumed existence of movement analyzers in the visual system which are selectively tuned to motion in a particular direction (Barlow \& Hill, 1963; Sekuler \& Pantle, 1967). According to this view, gazing for some time at a stimulus moving in one direction (the "inspection" or "adaptation" stimulus) fatigues the analyzers which are specifically tuned to that direction. Subsequently, when the observer gazes at a stationary pattern (the "test" stimulus), activity in the fatigued analyzers remains depressed, and, hence, there is relatively more activity in the analyzers tuned to the opposite direction. Perception is therefore of "opposite" movement.

This research was supported in part by NINCDS Grants NS12045 and NS05729. We are grateful to Lynn Halpern for her assistance in the planning and execution of these experiments. Some of the data were reported at the 50th meeting of the Midwestern Psychological Association, Chicago, May 1978. The current address of first author is: Parmly Hearing Institute, Loyola University of Chicago, 6525 N. Sheridan Road, Chicago, Illinois 60626.
This explanation of visual motion aftereffects enjoys strong physiological support, and, in fact, was originally prompted by the classic electrophysiological work of Hubel and Wiesel (1962). These investigators found single neurons in the cat's visual cortex which were specifically responsive to motion of a pattern in a particular direction. Barlow and Hill (1963) found such direction-specific units also in the rabbit's retina, and showed that the level of activity in these units declined with time of stimulation, remained suppressed for some time after stimulation ceased, then gradually rose back to baseline. This activity in single units is exactly parallel to the psychophysical effects of adaptation, aftereffect, and decay of aftereffect.

Direction-specific neurons have also been discovered in the auditory system. Altman and his colleagues have found single units in the cat's inferior colliculus and medial geniculate body which responded best to particular directions of movement of a sound source (simulated with headphone presentation) (Altman, 1968; Altman, Syka, \& Shmigidina, 1970). Sovijarvi and Hyvarinen (1974) found the same selectivity in single units of the cat's auditory cortex when the stimuli were real sound sources moving in the vertical and horizontal planes.

The parallels between the visual and auditory physiological data suggest that there may be similar mechanisms underlying motion perception in the two modalities. In particular, if there are direction-specific analyzers in the auditory system, and if auditory motion perception is mediated by the total activity of analyzers tuned to opposite directions, we would expect that auditory motion aftereffects might be demonstrated psychophysically. ${ }^{1}$ This paper reports a 
preliminary experiment which deals with possible aftereffects as a function of two variables: frequency of the stimulus and velocity of the auditory movement.

\section{METHOD}

\section{The Adaptation Stimulus}

To produce auditory movement, we presented special stimuli to listeners through headphones. These stimuli (an example is shown in Figure 1) were binaural tones with dynamically varying interaural temporal and interaural amplitude differences, producing for the listeners an intracranial acoustic image which appeared to move along a path between the ears. We chose the onset interaural differences to simulate the position of a real source situated in the horizontal plane $30 \mathrm{deg}$ off midline. ${ }^{2}$ The interaural differences decreased linearly from these values such that half way through the presentation there were no interaural differences (simulating a sound source straight ahead), and at stimulus offset the differences were equal, but opposite in sign to the onset interaural differences (simulating a sound source $30 \mathrm{deg}$ off midline to the opposite side). One "sweep" of the adaptation stimulus thus corresponds, in terms of the changing interaural differences, to the movement of a real sound source in the horizontal plane from $30 \mathrm{deg}$ on one side of the observer to $30 \mathrm{deg}$ on the other side.

The velocity of movement of the adaptation stimulus was manipulated by varying the duration of sweep: sweep durations of $2,000,1,000$, and $300 \mathrm{msec}$ corresponded, respectively, to adaptation velocities of 30,60 , and $200 \mathrm{deg} / \mathrm{sec}$ (this parameter will henceforth be referred to as velocity).

The adaptation stimulus was presented repeatedly, interrupted by 100 msec of silence between each "sweep." Thus, the auditory movement we simulate is a sequence of left-to-right (or right-toleft) sweeps separated by silence.

\section{Procedure}

We employed four types of adaptation stimulus, differing along dimensions of frequency and velocity of (simulated) movement. Three of the stimuli were $500-\mathrm{Hz}$ tones, presented at velocities of 30,60 , and $200 \mathrm{deg} / \mathrm{sec}$, respectively. The fourth was a $2,000-\mathrm{Hz}^{3}$ tone presented at a velocity of $200 \mathrm{deg} / \mathrm{sec}$. For each of these four adaptation stimuli, there were three possible modes of presentation: left-to-right sweep, right-to-left sweep, and a control condition that produced no illusion of movement. In this latter condition, tones were presented with no interaural differences; the acoustic image was that of a pulsing tone, the position of which remained fixed in the center of the head.

For maximum efficiency in data collection, we employed a probe technique to assess the effects of adaptation. With this procedure, the adaptation stimulus remains fixed through a block of trials, and therefore observers presumably remain in a "fully adapted state" while indicating their perceptions of various (probe) stimuli. During a single run (approximately $8 \mathrm{~min}$ ), one of the 12 adaptation stimuli ( 4 types $\times 3$ presentation modes) was presented continually. At $10-\mathrm{sec}$ intervals, the adaptation stimulus was interrupted for a 1-sec presentation of a test stimulus (the probe) to which the observer responded by pressing one of two buttons, indicating whether he felt the probe had moved "left" or "right" (observers were instructed to guess if unsure). There were seven different probe stimuli (to be described below), each of which was presented six times in random order. Thus, 42 responses were collected during a "run."

The probe was always a single, 1 -sec presentation of a tone of the same frequency as the adaptation stimulus. It was presented at one of seven velocities: $0 \mathrm{deg} / \mathrm{sec}$ (diotic) or 10,20 , or $40 \mathrm{deg} / \mathrm{sec}$, sweeping either left or right. As with the adaptation stimuli, the moving probe stimuli were synthesized to move sym-

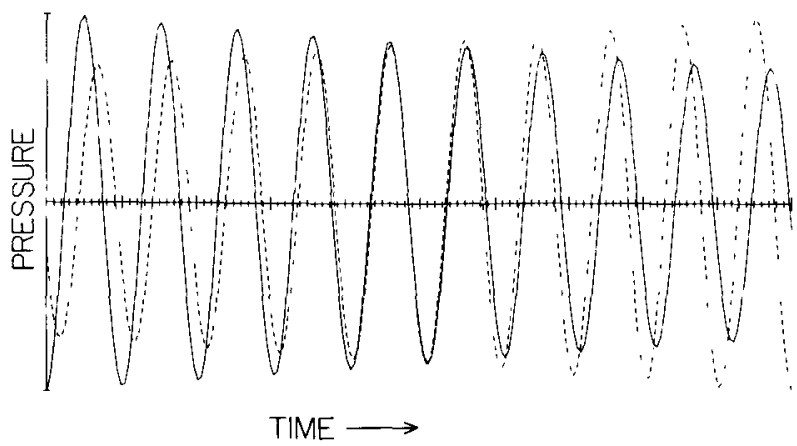

Figure 1. Representation of the binaural stimulus employed to simulate left-to-right movement. At stimulus onset, the waveform in the left ear (solid line) is more intense and leads, in time, the waveform in the right ear (dotted line). At stimulus of fset, these interaural relations are reversed.

metrically about midline. Velocity of the probe was manipulated, not by varying sweep duration as was done for the adaptation stimuli, but by varying the starting and ending positions of the simulated moving source $(20,10$, and $5 \mathrm{deg}$ off midline, respectively, for test velocities of 40,20 , and $10 \mathrm{deg} / \mathrm{sec}$ ). For each combination of adaptation stimulus and probe, 12-24 responses were collected (not all in a single session). The dependent variable was percent responses "left."

\section{Subjects and Apparatus}

Three female observers with clinically normal hearing were tested simultaneously in 102 -h sessions spread over a 2 -week period. At least $6 \mathrm{~h}$ of training were provided for each observer before data were collected. All experimentation was conducted in an IAC sound-insulated chamber. Stimuli were delivered through matched TDH -49 headphones at a comfortable listening level ( $75 \mathrm{~dB}$ SPL). Throughout a session, a continuous, low-level (60 dB SPL overall) $3,000-\mathrm{Hz}$ low-pass Gaussian noise was present; this noise was presented diotically (identically in the two ears) to provide a centered background image which observers could use for reference.

\section{RESULTS}

The data are plotted in Figures 2-5 for the four types of adaptation stimulus. In each figure, the percentage of responses "left" is plotted as a function of probe stimulus velocity for the three observers (shown separately in the three panels). The parameter is the "mode" of adaptation (leftward, rightward, or control).

The data from the control conditions suggest that, in the absence of direction-specific adaptation, the observers were generally unbiased in their responses to the test stimuli: for a stationary $(0 \mathrm{deg} / \mathrm{sec})$ probe, they responded "left" about $50 \%$ of the time. To the extent that there is a positive aftereffect, the function plotted for the case of "leftward" adaptation (triangles) should lie below and to the left of the control function (observers should tend to respond "left" less often), while the function for "rightward" adaptation (squares) should lie above and to the right of the control data (showing a tendency to respond 


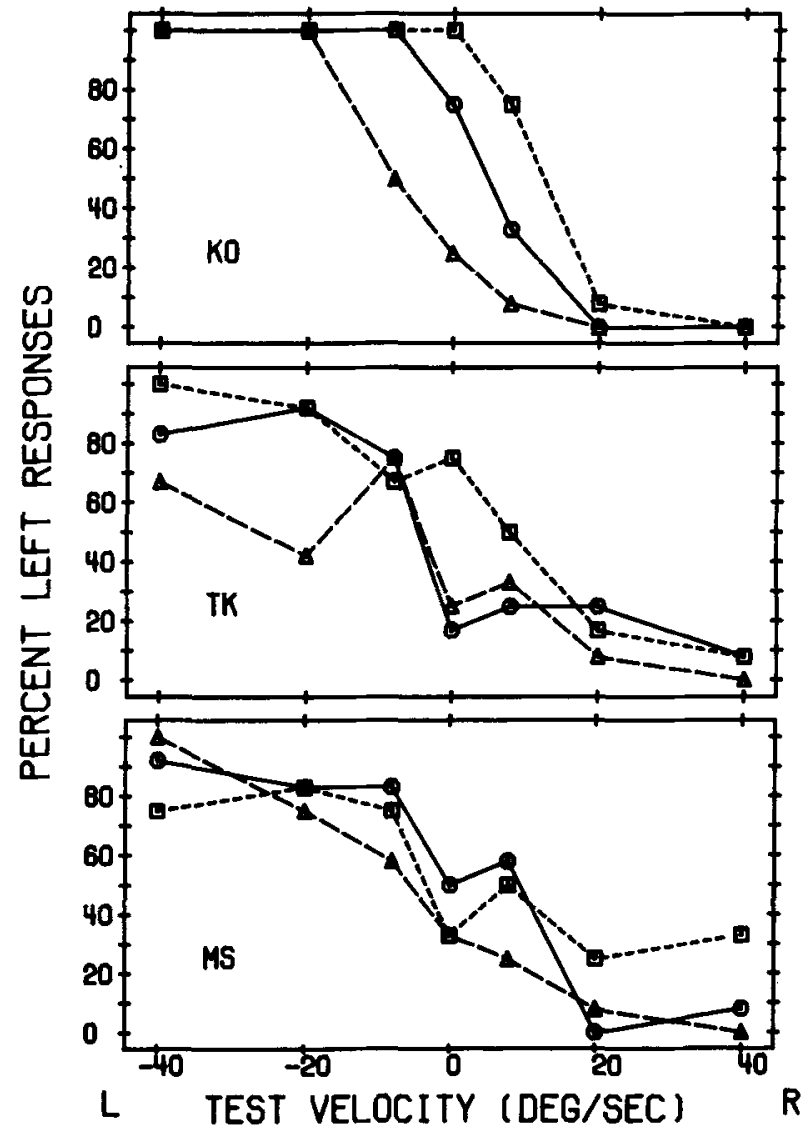

Figure 2. Data for three subjects. 500-Hz signal, 30-deg/sec adaptation velocity. Percent left responses is plotted as a function of test stimulus velocity for three conditions of adaptation: $\Delta$, leftward adaptation; $\square$, rightward adaptation; $O$, stationary adaptation (control).

"left" more often). When the adaptation functions coincide with the control function, there is no evidence for an auditory motion aftereffect.

Figures 2-4 plot the data obtained using the $500-\mathrm{Hz}$ adaptation stimuli with sweep velocities of 30,60 , and $200 \mathrm{deg} / \mathrm{sec}$, respectively. For the slowest velocity (Figure 2), only one listener (K.O.) showed clear evidence of adaptation, reflected in the relative positions of the response functions for the different directions of adaptation. As adaptation velocity increased (Figures 3-4), the aftereffect for this subject became more pronounced (the functions for "left" and "right" adaptation conditions became increasingly divergent), and an effect became apparent for the other two observers as well. At $500 \mathrm{~Hz}$, therefore, we may conclude that the magnitude of the aftereffect for our three listeners is an increasing function of adaptation stimulus velocity, over the range of velocities tested.

Figure 5 shows the data collected for the $2,000-\mathrm{Hz}$ adaptation stimulus presented at a velocity of $200 \mathrm{deg} / \mathrm{sec}$. At this higher frequency, there is little or no evidence for motion aftereffects. We should reemphasize at this point that the movement of the higher frequency adaptation stimulus was judged (by our listeners and by us) to be as salient as that of the lower frequency stimulus. This equivalence of movement perception across frequency is expected, based on subjects' equivalent performance in localizing real (stationary) sound sources of different frequencies. ${ }^{4}$ Yet, despite equal apparent movement of the adaptation stimuli in the low- and high-frequency conditions represented in Figures 4 and 5 , the motion aftereffect is substantially reduced or completely absent at the higher frequency. In other words, we see a definite frequency effect in the measurement of auditory aftereffects.

\section{DISCUSSION}

We conclude that auditory motion aftereffects can be demonstrated psychophysically if the right combination of stimulus parameters is used. Specifically, adaptation with a $500-\mathrm{Hz}$ tone sweeping repeatedly in one direction at a velocity of $200 \mathrm{deg} / \mathrm{sec}$ produced

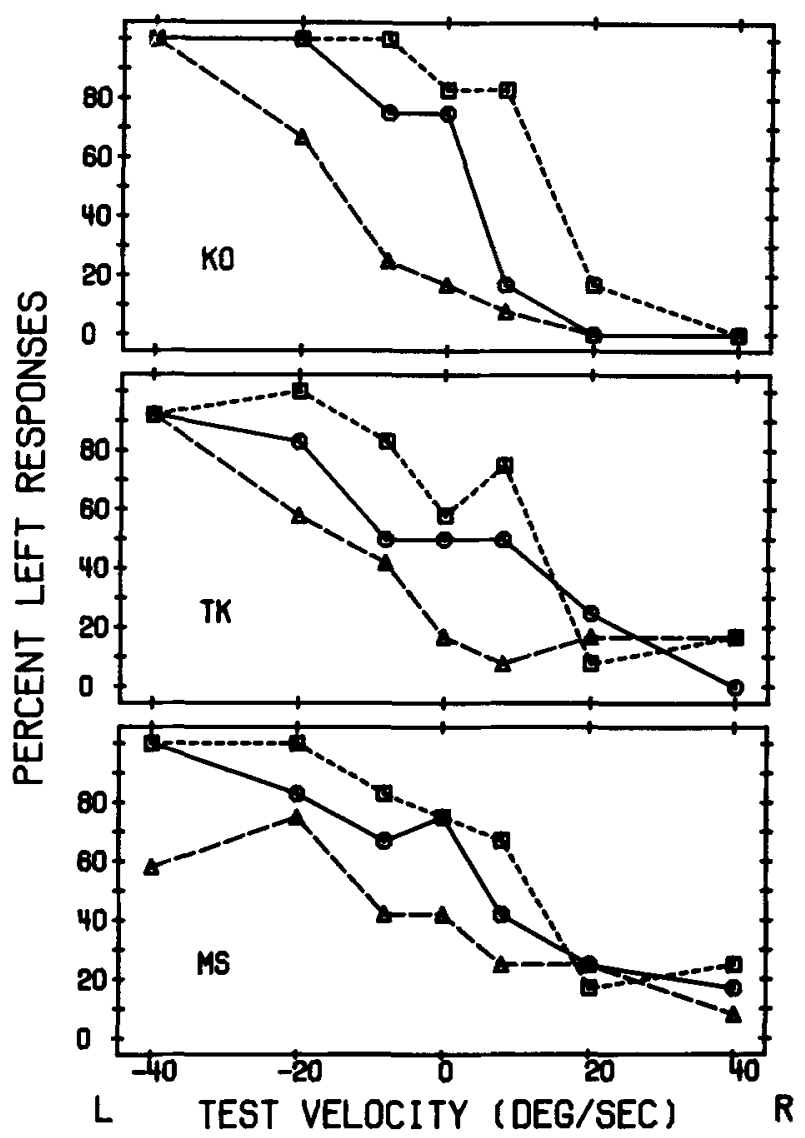

Figure 3. Data for three subjects. 500-Hz signal, 60-deg/sec adaptation velocity. See caption for Figure 2. 


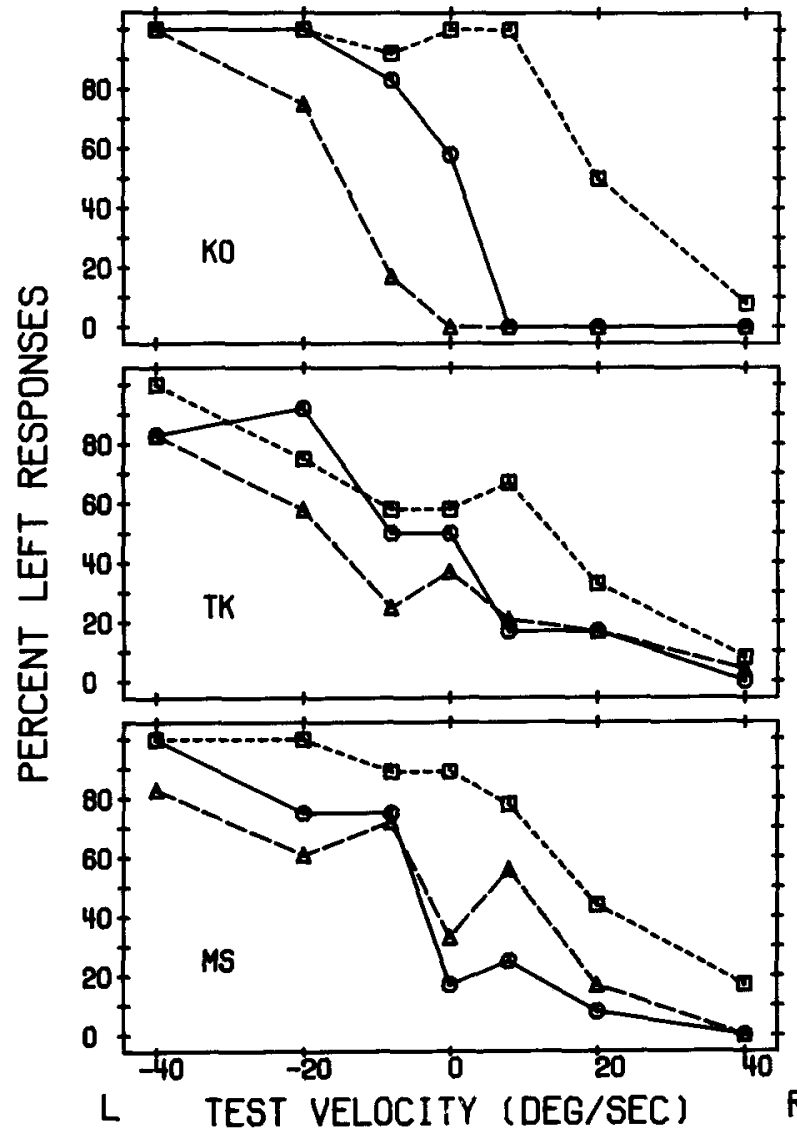

Figure 4. Data for three subjects. 500-Hz signal, 200-deg/sec adaptation velocity. See caption for Figure 2.

a consistent tendency in all observers to judge stationary stimuli as moving in the opposite direction. Furthermore, as has been demonstrated for visual motion aftereffects (Scott, Jordan, \& Powell, 1963), the effect of auditory adaptation was found to combine algebraically with real movement in probe stimuli, such that the perception of motion was either canceled or enhanced, depending on whether the movement of the probe was in the opposite or same direction as the movement of the adaptation stimulus.

We might consider as a working hypothesis that the widely accepted interpretation of the visual "waterfall effect" might be applied to our auditory data as well. Thus, the perception of moving sound sources is hypothesized to be mediated by directionspecific motion analyzers in the auditory system (the physiological data cited earlier would place the analyzers in the inferior colliculus and/or medial geniculate body). The motion aftereffect reflects that activity in the set of analyzers tuned to one particular direction is temporarily depressed (fatigued by the previous exposure to movement), allowing the relative activity of the analyzers tuned in the opposite direction to dominate during a neutral stimulus con- dition (nonmovement). The fact that the magnitude of aftereffect depends on the velocity of motion would reflect the distribution of velocities "preferred" by the population of analyzers: thus, at $500 \mathrm{~Hz}$, as the stimulus velocity decreases, it is "preferred" by fewer and fewer analyzers, and the magnitude of the aftereffect should diminish. Finally, the fact that aftereffects are frequency-specific might indicate that the distribution of velocity "preferences" of the analyzers depends on the stimulus frequency. Our failure to obtain an aftereffect at $2,000 \mathrm{~Hz}$ may reflect that, of the subpopulation of movement analyzers tuned to $2,000 \mathrm{~Hz}$, few or none "preferred" a velocity of $200 \mathrm{deg} / \mathrm{sec}$. At this higher frequency, a slower velocity stimulus might have elicited the aftereffect (unfortunately, we employed only one velocity at $2,000 \mathrm{~Hz}$ ).

This account of auditory aftereffects, borrowed in toto from the vision literature, is of course entirely speculative in the present context, and should be regarded cautiously. We must stress that, while the visual motion ("waterfall") aftereffect is quite a robust phenomenon, easily demonstrated over a wide range of stimulus conditions, the auditory analog

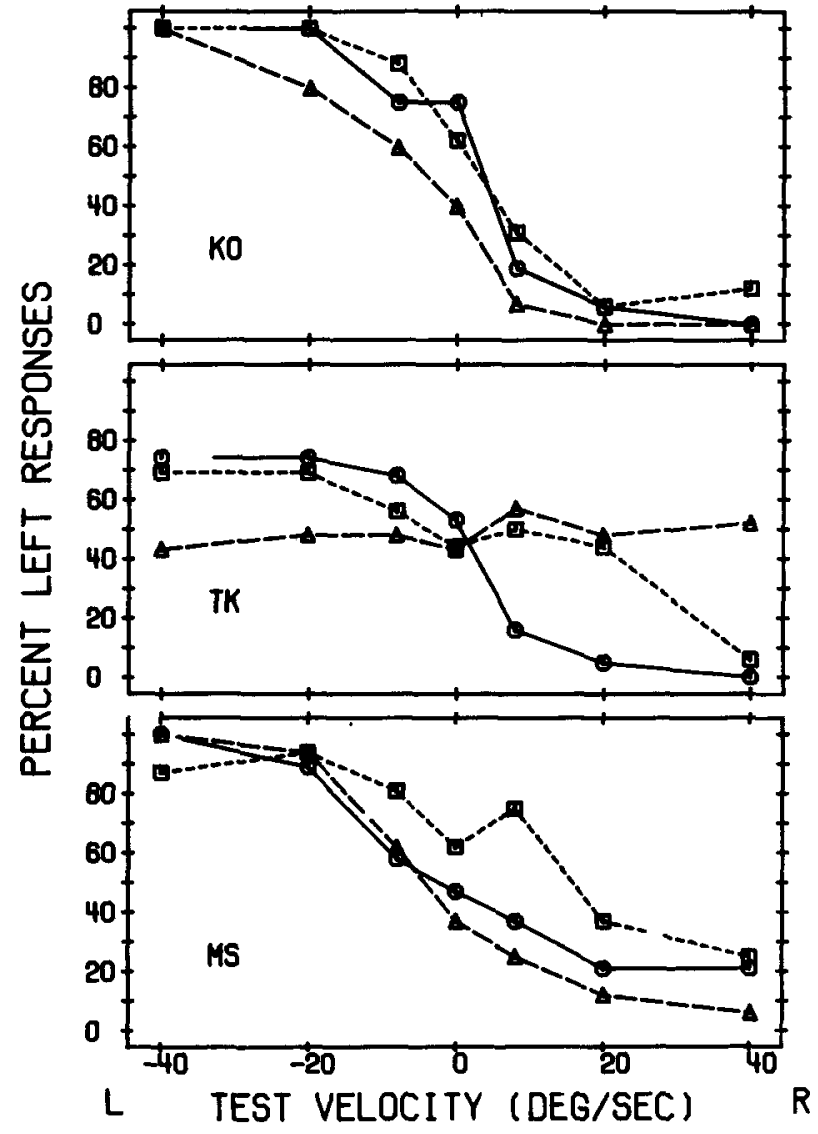

Figure 5. Data for three subjects. 2,000-Hz signal, 200-deg/sec adaptation velocity. See caption for Figure 2. 
that we have measured is relatively subtle, apparently demonstrable only in relatively few circumstances. This great difference in the strength of the visual and auditory aftereffects is illustrated in the methods used to measure them: While duration of apparent motion is the typical measure of the strength of visual aftereffects, the auditory aftereffect was much too transitory to allow such a measurement (all auditory aftereffects disappeared within 1-3 sec). Instead, we had to employ a two-alternative ("right"-"left") forced-choice paradigm to reveal the existence of the auditory effect.

Given that auditory motion aftereffects can be demonstrated with appropriate stimulus conditions, we may speculate as to whether the effect is truly an auditory phenomenon, or is mediated by the visual, kinesthetic, and/or proprioceptive systems. In other words, does the perception of auditory motion during the adaptation period affect other systems (visual orientation, musculature of the eyes, postural attitude, etc.), such that the reported movement of the auditory probe stimuli is due to some kind of adaptation in these systems, and not specifically to auditory adaptation? Along these same lines, we might ask whether the auditory motion aftereffect is a sensory effect at all, as opposed to a nonsensory phenomenon (e.g., a change in judgmental bias or adaptation level).

There are several features of our data which suggest that the obtained aftereffect is indeed a purely auditory phenomenon. That it is not mediated by the visual system is clear, since the temporal parameters in the two cases are not of the same order of magnitude. Note that we found best auditory aftereffects for adaptation velocities of $200 \mathrm{deg} / \mathrm{sec}$. The best visual aftereffects are obtained with adaptation velocities of 4-6 deg/sec (Sekuler \& Ganz, 1963). Therefore, the auditory effect is not a simple translation of 'auditory stimulation into visual space.

More generally, the "frequency effect" observed in our data (see Figures 4-5) suggests that the auditory motion aftereffect is not mediated by another system (sensory or nonsensory), but that its basis is purely auditory. In other words, since the frequency content of the stimulus is critical in determining whether or not the auditory aftereffect occurs, any intervening system which would mediate the effect would have to be frequency-specific. Rather than implicate some other frequency-dependent system as a mediator, it seems most parsimonious to conclude that the auditory system itself is directly affected by the prolonged motion stimulation, resulting in the effects we have measured.

\section{SUMMARY AND CONCLUSIONS}

With appropriately chosen stimulus conditions, observers' judgments of auditory motion in the hori- zontal plane are clearly and consistently affected by their immediately previous experience with auditory movement. The fact that auditory motion aftereffects are frequency-specific (they occur at $500 \mathrm{~Hz}$, but not at $2,000 \mathrm{~Hz}$ ) suggests that the effects are specific to the auditory system (i.e., they are not likely mediated by another sensory system, nor are they likely based on judgmental criterion shifts).

Although the auditory motion aftereffect is weaker than the "waterfall" effect in vision, we feel that the parallel results suggest that analogous mechanisms underlie motion perception in the two modalities. In particular, auditory motion perception might be mediated by direction-specific movement analyzers in the auditory system. This interpretation would be congruous with the auditory neurophysiological data cited earlier, in which single units were reported to respond selectively to motion in one particular direction. Additional psychophysical work, focusing on stimulus frequency, velocity, and the manner in which auditory motion is produced, will hopefully reveal whether the analog between visual and auditory motion perception will ultimately prove useful.

\section{REFERENCES}

Altman, J. A. Are there neurons detecting direction of sound source motion? Experimental Neurology, 1968, 22, 13-25.

Altman, J. A., Syka, J., \& Shmigidina, G. N. Neuronal activity in the medial geniculate body of the cat during monaural and binaural stimulation. Experimental Brain Research, 1970, 10, 81-93.

Barlow, H. B., \& Hill, R. M. Evidence for a physiological explanation of the waterfall phenomenon and figural aftereffects. Nature, 1963, 200, 1345-1347.

Ehrenstein, W. H. Direction-specific acoustical aftereffects. Journal of the Acoustical Society of America, 1978, 64, S35(A).

Fedderson, W. E., Sandel, T. T., Teas, D. C., \& Jeffress, L.A. Localization of high-frequency tones. Journal of the Acoustical Society of America, 1957, 29, 988-991.

Gates, L. W. The after-effect of visually observed movement. American Journal of Psychology, 1934, 46, 34-46.

Harris, J. D., \& Se rge ANT, R. L. Monaural/binaural minimum audible angles for a moving sound source. Journal of Speech \& Hearing Research, 1971, 14, 618-629.

Hubel, D. H., \& WiEsEL, T. N. Receptive fields, binocular interaction, and functional architecture in the cat's visual cortex. Journal of Physiology, 1962, 160, 106-154.

Milus, A. W. On the minimum audible angle. Journal of the Acoustical Society of America, 1958, 30, 237-246.

Molino, J. A. Psychophysical verification of predicted interaural differences in localizing distant sound sources. Journal of the Acoustical Society of America, 1974, 55, 139-147.

Sandel, T. T., Teas, D. C., Fedderson, W. G., \& Jeffress, L. A. Localization of sound from single and paired sources. Journal of the Acoustical Society of America, 1955, 27, 842-852.

Scott, T. R., Jordan, A. E., \& Powell, D. A. Does visual aftereffect of motion add algebraically to objective motion of the test stimulus? Journal of Experimental Psychology, 1963, 66, 500-505.

Sekule R, R. W., Ganz, L. Aftereffect of seen motion with a stabilized retinal image. Science, 1963, 139, 419-420. 
Sekuler, R., \& Pantle, A. A model for after-effects of seen movement. Vision Research, 1967, 7, 427-439.

Sovijarvi, A. R. A., \& Hyvarinen, J. Auditory cortical neurons in the cat sensitive to the direction of sound source movement. Brain Research, 1974, 73, 455-471.

Stevens, S. S., \& Newman, E. B. The localization of actual sources of sound. American Journal of Psychology, 1936, 48, 297-306.

Wolgemuth, A. On the after-effect of seen movement. British Journal of Psychology, 1911, Monograph Supplement 1, 1-117.

\section{NOTES}

1. Ehrenstein (1978) has recently reported that exposure to auditory movement affects an observer's subsequent localization of a stationary auditory object: in particular, the apparent position of a source is displaced about $2 \mathrm{deg}$ to the side opposite the direction of movement of a previous adaptation stimulus. Although this "auditory displacement aftereffect" is relevant to mechanisms underlying our perception of auditory (static) space, if direction-specific motion analyzers exist, we would predict that a stationary test stimulus (presented after adaptation to movement), should not only be displaced, but should appear to move. To date there have been no reports of such an auditory motion aftereffect.
2. From a sound source in the horizontal plane located $30 \mathrm{deg}$ off midline, the wavefront arrives at the nearer ear about $.25 \mathrm{msec}$ before it arrives to the farther ear (independent of frequency). The interaural intensity difference of the sound from this source depends on its frequency content: for a $500-\mathrm{Hz}$ tone, the difference is about $3 \mathrm{~dB}$; for a $2,000-\mathrm{Hz}$ tone, the difference is about $7 \mathrm{~dB}$. These measurements have been made by Fedderson, Sandel, Teas, and Jeffress (1957).

3. By employing appropriate interaural parameters, we were able to produce movement at $2,000 \mathrm{~Hz}$ which was as salient as that at $500 \mathrm{~Hz}$ (see Footnote 2). Thus, in terms of the perception of movement of the adaptation stimulus, the low-frequency and high-frequency conditions were equivalent.

4. Although the classic paper by Stevens and Newman (1936) reported that tones around $2,000-4,000 \mathrm{~Hz}$ are localized less precisely than tones of lower (or higher) frequency, more recent studies have revealed that this frequency effect is small or negligible, especially in the region around midline (Harris \& Sergeant, 1971; Mills, 1958; Molino, 1974; Sandel, Teas, Fedderson, \& Jeffress, 1955).

(Received for publication April 12, 1979; revision accepted September 21, 1979.) 